Article

\title{
Fixed Point Results for Multi-Valued Contractions in $b$-Metric Spaces and an Application
}

\author{
Haitham Qawaqneh ${ }^{1, *,+}$, Mohd Salmi Md Noorani ${ }^{1, \dagger}$, Wasfi Shatanawi ${ }^{2,3, \dagger}$, Hassen Aydi ${ }^{4,+}+()$ \\ and Habes Alsamir ${ }^{1,+}$ \\ 1 Department of Mathematics, Faculty of Science and Technology, University Kebangsaan Malaysia, UKM \\ Bangi, Selangor DE 43600, Malaysia; msn@ukm.my (M.S.M.N.); h.alsamer@gmail.com (H.A.) \\ 2 Department of Mathematics and General Courses, Prince Sultan University, Riyadh 11586, Saudi Arabia; \\ wshatanawi@psu.edu.sa \\ 3 Department of Medical Research, China Medical University Hospital, China Medical University, \\ Taichung 40402, Taiwan \\ 4 Department of Mathematics, College of Education in Jubail, Imam Abdulrahman Bin Faisal University, P.O. \\ Box 12020, Industrial Jubail 31961, Saudi Arabia; hmaydi@iau.edu.sa or hasssen.aydi@isima.rnu.tn \\ * Correspondence: Haitham.math77@gmail.com; Tel.: +60128813450 \\ + These authors contributed equipollently to this work.
}

Received: 6 December 2018; Accepted: 16 January 2019; Published: 1 February 2019

\begin{abstract}
In this paper, by characterizing a weak contractive condition based on using $C$-functions and $\alpha$-admissible multi-valued mapping of type $S$, we present some fixed point results for $(\alpha, F)$-admissible multi-valued mappings in the setting of $b$-metric spaces. Some examples and an application are added in order to show the reliability of our obtained results. Our results amend, unify, and generalize some existing results in the literature. The scientific novelty of our main results is to take new contraction self-mappings in $b$-metric spaces for multi-valued mappings.
\end{abstract}

Keywords: $\alpha$-admissible mapping of type $S$; multi-valued mapping; Fixed point; $b$-metric space; Hausdorff metric

\section{Introduction}

The Banach contraction principle is considered to be one of the most useful tools in fixed point theory. It has been extended or generalized in different directions and many (common) fixed point theorems have been provided (see References [1-13]). The theory of fixed points for multi-valued mappings has been developed after the famous paper of Nadler [14]. Several authors have given many forms of multi-valued mapping conditions by using the concept of Hausdorff-Pompieu metric. Among them, we cite References [15-17].

First, we recite some notations, definitions, and elementary results needed in the sequel. Denote by $\mathbb{N}$ and $\mathbb{R}$ the sets of positive integers and real numbers, respectively. Consider a metric space $(X, d)$. Denote by $2^{X}$ (resp. $C L(X)$ ) the family of subsets (resp. of closed subsets) of $X$. Let $C B(X)$ be the class of all nonempty closed bounded subsets of $X$ and $F(f)$ be the set of all fixed points of $f$.

Definition 1 ([14]). A point $u \in X$ is called a fixed point of the multi-valued map $T: X \rightarrow 2^{X}$ if $u \in T u$.

For $B, C \in C L(X)$, let $H: C L(X) \times C L(X) \rightarrow[0, \infty]$ be defined by

$$
H(B, C)= \begin{cases}\max \{\delta(B, C), \delta(C, B)\}, & \text { if the maximumexists } \\ \infty, & \text { otherwise, }\end{cases}
$$


where $\delta(B, C)=: \sup _{a \in B} d_{b}(b, C)$. Such $H$ is called the generalized Hausdorff-Pompieu metric induced by the metric $d$. The known result of Nadler [14] is as follows:

Definition 2 ([14]). Let $(X, d)$ be a metric space. A map $f: X \rightarrow C B(X)$ is said to be a multi-valued contraction if there exists $0 \leq \lambda<1$ such that

$$
H(f x, f y) \leq \lambda d(x, y)
$$

for all $x, y \in X$.

Bakhtin [18] and Czerwik [19] initiated the concept of a $b$-metric.

Definition $3([18,19])$. Let $X$ be a nonempty set and $s \geq 1$ be a given real number. Take $d_{b}: X \times X \rightarrow[0, \infty)$. Suppose that for all $\zeta, \eta, \xi \in X$, we have:

(i) $d_{b}(\zeta, \eta)=0$ if and only if $\zeta=\eta$;

(ii) $d_{b}(\zeta, \eta)=d_{b}(\eta, \zeta)$;

(iii) $d_{b}(\zeta, \xi) \leq s\left[d_{b}(\zeta, \eta)+d_{b}(\eta, \xi)\right]$.

Then, $d_{b}$ is said a b-metric and the triplet $\left(X, d_{b}, s\right)$ is called $a b$-metric space.

The authors in References [18,19] extended the Banach contraction principle to the class of $b$-metric spaces. For other results in the setting of $b$-metric spaces, see References $[3,4,10,20-23]$.

Example 1 ([18,19]). Consider the set $X=[0,1]$ endowed with the function $d_{b}: X \times X \rightarrow[0, \infty)$ defined by $d_{b}(\zeta, \eta)=|\zeta-\eta|^{2}$ for all $\zeta, \eta \in X$. Here, $\left(X, d_{b}, 2\right)$ is a $b$-metric space, but it is not a metric space.

Let $\left(X, d_{b}, s\right)$ be a $b$-metric space. Let $\left\{\theta_{n}\right\}$ be a sequence in $X$.

(i) $\left\{\theta_{n}\right\} \subseteq X$ converges to a point $x \in X$ if $\lim _{n \rightarrow \infty} d_{b}\left(\theta_{n}, x\right)=0$,

(ii) $\left\{\theta_{n}\right\} \subseteq X$ is Cauchy if, for each $\epsilon>0$, there is some $n(\epsilon) \in \mathbb{N}$ such that $d_{b}\left(\theta_{n}, \theta_{m}\right)<\epsilon$ for all $m, n \geq n(\epsilon)$

(iii) $\left(X, d_{b}, s\right)$ is said complete if any Cauchy sequence is convergent in $X$.

Lemma 1 ([2]). Let $\left(X, d_{b}, s\right)$ be $a b$-metric space with $s \geq 1$. Suppose that $\left\{\theta_{n}\right\}$ and $\left\{\eta_{n}\right\}$ are $b$-convergent sequences to $\theta$ and $\eta$, respectively. Then,

$$
\frac{1}{s} d_{b}(\theta, \eta) \leq \lim _{n \rightarrow \infty} \inf d_{b}\left(\theta_{n}, \eta_{n}\right) \leq \lim _{n \rightarrow \infty} \sup d_{b}\left(\theta_{n}, \eta_{n}\right) \leq s^{2} d_{b}(\theta, \eta) .
$$

In the case that $\theta=\eta$, we get $\lim _{n \rightarrow \infty} d_{b}\left(\theta_{n}, \eta_{n}\right)=0$. Moreover, for each $\eta \in X$,

$$
\frac{1}{s} d_{b}(\theta, \eta) \leq \lim _{n \rightarrow \infty} \inf d_{b}\left(\theta_{n}, \eta\right) \leq \lim _{n \rightarrow \infty} \sup d_{b}\left(\theta_{n}, \eta\right) \leq s^{2} d_{b}(\theta, \eta) .
$$

Lemma 2 ([2]). Let $\left(X, d_{b}, s\right)$ be a $b$-metric space with $s \geq 1$. Let $\left\{\theta_{n}\right\}$ be a sequence in $X$ such that

$$
\lim _{n \rightarrow \infty} d_{b}\left(\theta_{n}, \theta_{n+1}\right)=0 .
$$


If $\left\{\theta_{n}\right\}$ is not $a b$-Cauchy sequence, then there exist $\epsilon>0$ and $\left\{\theta_{m(k)}\right\}$ and $\left\{\theta_{n(k)}\right\}$ two sequences of positive integers such that

$$
\begin{array}{lcc}
\epsilon \leq \lim _{n \rightarrow \infty} \inf d_{b}\left(\theta_{m(k)}, \theta_{n(k)}\right) \leq \lim _{n \rightarrow \infty} \sup d_{b}\left(\theta_{m(k)}, \theta_{n(k)}\right) & \leq s \epsilon \\
\frac{\epsilon}{s} \leq \lim _{n \rightarrow \infty} \inf d_{b}\left(\theta_{m(k)}, \theta_{n(k)+1}\right) \leq \lim _{n \rightarrow \infty} \sup d_{b}\left(\theta_{m(k)}, \theta_{n(k)+1}\right) & \leq s^{2} \epsilon \\
\frac{\epsilon}{s} \leq \lim _{n \rightarrow \infty} \inf d_{b}\left(\theta_{m(k)+1}, \theta_{n(k)}\right) \leq \lim _{n \rightarrow \infty} \sup d_{b}\left(\theta_{m(k)+1}, \theta_{n(k)}\right) & \leq s^{2} \epsilon \\
\frac{\epsilon}{s^{2}} \leq \lim _{n \rightarrow \infty} \inf d_{b}\left(\theta_{m(k)+1}, \theta_{n(k)+1}\right) \leq \lim _{n \rightarrow \infty} \sup d_{b}\left(\theta_{m(k)+1}, \theta_{n(k)+1}\right) \leq s^{3} \epsilon .
\end{array}
$$

Lemma 3 ([21]). Let $\left(X, d_{b}, s\right)$ be a $b$-metric space. For all $P, Q, R \in C B(X)$, and $\xi, \theta \in X$, we have:

(i) $d_{b}(\xi, Q) \leq d_{b}(\xi, \eta)$ for any $\eta \in Q$;

(ii) $\quad d_{b}(P, Q) \leq H(P, Q)$;

(iii) $\delta(P, Q) \leq H(P, Q)$;

(iv) $d_{b}(\xi, Q) \leq H(P, Q)$ for any $\xi \in P$;

(iiv) $H(P, P)=0$;

(vi) $H(P, Q)=H(Q, P)$;

(vii) $H(P, R) \leq s(H(P, Q)+H(Q, R))$;

(viii) $d_{b}(\xi, P) \leq s\left(d_{b}(\xi, \theta)+d_{b}(\theta, P)\right)$.

Remark 1 ([21]). Let $\left(X, d_{b}, s\right)$ be a $b$-metric space. The function $H: C L(X) \times C L(X) \rightarrow[0, \infty)$ is a generalized Hausdorff $b$-metric, that is, $H(A, B)=+\infty$ if $\max \{\delta(A, B), \delta(B, A)\}$ does not exist.

Lemma 4 ([21]). Let $\left(X, d_{b}, s\right)$ be a b-metric space. For $P \in C L(X)$ and $\xi \in X$, we have

$$
d_{b}(\xi, P)=0 \Leftrightarrow \xi \in \bar{P}=P,
$$

where the closure of the set $A$ is denoted by $\bar{A}$.

The concept of $\alpha$-admissibility was first initiated by Samet et al. [24].

Definition 4 ([24]). Given $f: X \rightarrow X$ and $\alpha: X \times X \rightarrow[0, \infty)$. $f$ is called $\alpha$-admissible if $\alpha(\zeta, \eta) \geq 1$ implies that $\alpha(f \zeta, f \eta) \geq 1$.

This concept was generalized by Sintunavarat [25].

Definition 5 ([25]). Let $X$ be a nonempty set. Let $f: X \rightarrow X$ and $\alpha: X \times X \rightarrow[0, \infty)$ be two mappings. Let $s \geq 1$ be a given real number. We say that $f$ is weak $\alpha$-admissible of type $S$ if for $x \in X$ and $\alpha(x, f x) \geq s$, then $\alpha(f x, f f x) \geq s$.

Definition 6 ([26]). Let $X$ be a nonempty set. Given $f: X \rightarrow C L(X)$ and $\alpha: X \times X \rightarrow[0, \infty)$. Then, $f$ is said to be $\alpha$-admissible whenever, for each $\xi \in X$ and $\eta \in f \xi$ with $\alpha(\xi, \eta) \geq 1$, we have $\alpha(\eta, \theta) \geq 1$ for each $\theta \in f \eta$.

After that, many authors used the concept of $\alpha$-admissible contractive type mappings to investigate the existence of fixed points (see References $[10,23,27-31]$ and references cited therein).

In 2014, Ansari [32] defined the concept of C-class functions.

Definition 7 ([32]). A mapping $F:[0, \infty) \times[0, \infty) \rightarrow \mathbb{R}$ is called a $C$-class function if it is continuous and for $s, t \in[0, \infty), F$ satisfies the following two conditions:

(a) $F(r, t) \leq r$; 
(b) $F(r, t)=r$ implies that either $r=0$ or $t=0$.

The family of $\mathcal{C}$-class functions is denoted by $\mathcal{C}$.

Example 2 ([32]). The following functions $F: \mathbb{R}^{+} \times \mathbb{R}^{+} \rightarrow \mathbb{R}$ are elements in $\mathcal{C}$ :

(a) $F(r, t)=r-t$ for all $r, t \in[0, \infty)$;

(b) $F(r, t)=k r$ for all $r, t \in[0, \infty)$, where $0<k<1$;

(c) $F(r, t)=r \beta(r)$ for all $r, t \in[0, \infty)$, where $\beta:[0, \infty) \rightarrow[0,1)$ is continuous;

(d) $F(r, t)=r-\varphi(r)$ for all $r, t \in[0, \infty)$, where $\varphi:[0, \infty) \rightarrow[0, \infty)$ is a continuous function such that $\varphi(t)=0$ if and only if $t=0$;

(e) $F(s, t)=\frac{s}{(1+t)^{a}}$ with $a \in(0, \infty)$, for all $r, t \in[0, \infty)$;

(f) $F(s, t)=\ln \left(t+k^{s}\right) /(1+t)$ with $k>1$, for all $r, t \in[0, \infty)$.

The generalization of the well-known Banach fixed point theorem with rational expressions was considered by Reference [33]. On the other hand, there are many works on fixed point theory using control functions (see References [24,28,34-37]). Let $\Phi$ be the set of lower semi-continuous functions $\phi:[0, \infty) \rightarrow[0, \infty)$ such that $\phi(t)=0 \Leftrightarrow t=0$. Let $\Psi$ be the set of nondecreasing functions $\psi:[0, \infty) \rightarrow[0, \infty)$ such that

$$
\sum_{n=1}^{+\infty} s^{n} \psi^{n}(t)<+\infty \text { for each } t>0,
$$

where $\psi^{n}$ is the $n^{\text {th }}$ iterate of $\psi$ and $s \geq 1$ is the coefficient of the b-metric space $(X, d)$.

Note that if $\psi \in \Psi$, we have $\lim _{n \rightarrow+\infty} \psi^{n}(t)=0, \psi(t)<\frac{t}{s} \leq t$ for each $t>0$ and $\psi(0)=0$.

Let $X$ be a $b$-metric space with coefficient $s \geq 1$ and $f: X \rightarrow 2^{X}$ be a multi-valued map. The graph of $f$ is given as

$$
\operatorname{Gr}(f)=\left\{(\xi, \eta) \in X^{2}, \eta \in f \xi\right\} .
$$

Such mapping $f$ is called upper semi-continuous if the inverse image of closed sets is closed.

In this paper, inserting $C$-class functions we present some fixed point results in the setting of a $b$-metric space for $\alpha$-admissible multi-valued type mappings of type $S$. An example and application are given.

\section{Main Results}

First, we introduce the notion of $\alpha$-admissible multi-valued mappings of type $S$.

Definition 8. Let $X$ be a nonempty set. Given $f: X \rightarrow C L(X)$ and $\alpha: X \times X \rightarrow[0,+\infty)$. Let $s \geq 1$ be a given real number. Such that $f$ is said to be $\alpha$-admissible of type $S$ if for each $\xi \in X$ and $\eta \in f \xi$ with $\alpha(\xi, \eta) \geq s$, we have $\alpha(\eta, \theta) \geq$ s for each $\theta \in f \eta$.

Example 3. Let $X=[-1,1]$ be endowed with the $b$-metric $d_{b}(\xi, \eta)=|\xi-\eta|^{2}$ with $s=2$ for all $\xi, \eta \in X$. Define $\alpha: X \times X \rightarrow[0, \infty)$ and $f: X \rightarrow C L(X)$ by

$$
\begin{gathered}
f \xi= \begin{cases}\{0,1\} & \text { if } \xi=-1, \\
\{1\} & \text { if } \xi=0, \\
\{-\xi\} & \text { if } \xi \notin\{-1,0\} .\end{cases} \\
\alpha(\xi, \eta)= \begin{cases}2 e^{\tilde{\zeta}+\eta} & \text { if } \xi=\eta, \\
4 & \text { if } \xi \neq \eta .\end{cases}
\end{gathered}
$$

It is easy show that $f$ is $\alpha$-admissible of type $S$. 
Example 4. Let $X=\mathbb{N} \cup\{0\}$ be endowed with the $b$-metric $d_{b}(\xi, \eta)=|\xi-\eta|^{2}$ for all $\xi, \eta \in X$ with $s=2$. Define the mappings $\alpha: X \times X \rightarrow[0, \infty)$ and $f: X \rightarrow C L(X)$ by

$$
\begin{gathered}
f \xi= \begin{cases}\{0,1\} & \text { if } \xi=0,1, \\
\{\xi, \xi+1\} & \text { if } \xi>1 .\end{cases} \\
\alpha(\xi, \eta)= \begin{cases}4 & \text { if } \xi, \eta \in\{0,1\}, \\
e^{\xi+\eta} & \text { if } \xi, \eta>1, \\
0 & \text { otherwise. }\end{cases}
\end{gathered}
$$

The mapping $f$ is $\alpha$-admissible of type $S$.

Example 5. Let $X=[0, \infty)$ be endowed with the $b$-metric $d_{b}(\xi, \eta)=|\xi-\eta|^{2}$ (with coefficient $s=2$ ) for all $\xi, \eta \in X$. Define $f: X \rightarrow C L(X)$ and $\alpha: X \rightarrow[0, \infty)$ by

$$
\begin{aligned}
& f \xi= \begin{cases}{\left[2,2+\frac{\xi}{3}\right]} & \text { if } \xi \in[0,1], \\
{[5,5+5 \xi]} & \text { if } \xi \in(1, \infty)\end{cases} \\
& \alpha(\xi, \eta)= \begin{cases}4 & \text { if } \xi, \eta \in[0,1] \\
e^{\tilde{\xi}+\eta} & \text { otherwise. }\end{cases}
\end{aligned}
$$

Clearly $f$ is $\alpha$-admissible of type $S$.

Definition 9. Let $\left(X, d_{b}, s\right)$ be $a b$-metric space with constant $s \geq 1$ and $f: X \rightarrow C L(X)$. Such that $f$ is an $(\alpha, F)$-multi-valued mapping if there exist $\alpha: X \times X \rightarrow[0, \infty), F \in \mathcal{C}, \psi \in \Psi$ and $\phi \in \Phi$ such that

$$
x, y \in X \text { with } \alpha(x, y) \geq s \Rightarrow H(f x, f y) \leq F(\psi(M(x, y)), \varphi(M(x, y))),
$$

where

$$
M(x, y)=\max \left\{d_{b}(x, y), \frac{d_{b}(x, f x)}{1+d_{b}(x, f x)}, \frac{d_{b}(y, f y)}{1+d_{b}(y, f y)}, \frac{1}{2 s}\left[d_{b}(x, f y)+d_{b}(y, f x)\right]\right\} .
$$

Theorem 1. Let $\left(X, d_{b}, s\right)$ be a $b$-metric space with constant $s \geq 1$ and $f: X \rightarrow C L(X)$ be an $(\alpha, F)$-admissible multi-valued mapping. Assume that:

(i) $f$ is $\alpha$-admissible of type $S$;

(ii) there exist $\theta_{0} \in X$ and $\theta_{1} \in f \theta_{0}$ such that $\alpha\left(\theta_{0}, \theta_{1}\right) \geq s$;

(iii) $\mathrm{Gr}(f)$ is a closed subset of $X^{2}$.

Then, $f$ has a fixed point.

Proof. By Condition (ii), $\theta_{0} \in X$ and $\theta_{1} \in f \theta_{0}$ such that $\alpha\left(\theta_{0}, \theta_{1}\right) \geq$ s. If $\theta_{0}=\theta_{1}$ or $\theta_{1} \in f \theta_{1}$, we deduce that $\theta_{1}$ is a fixed point of $f$ and so the proof is done. Now, we assume that $\theta_{0} \neq \theta_{1}$ and $\theta_{1} \notin f \theta_{1}$. By Lemma $4, d_{b}\left(\theta_{1}, f \theta_{1}\right)>0$. Applying Equation (3), we have

$$
\begin{aligned}
0<d_{b}\left(\theta_{1}, f \theta_{1}\right) & \leq H\left(f \theta_{0}, f \theta_{1}\right) \\
& \leq F\left(\psi\left(M\left(\theta_{0}, \theta_{1}\right)\right), \phi\left(M\left(\theta_{0}, \theta_{1}\right)\right)\right),
\end{aligned}
$$


where

$$
\begin{aligned}
M\left(\theta_{0}, \theta_{1}\right) & =\max \left\{d_{b}\left(\theta_{0}, \theta_{1}\right), \frac{d_{b}\left(\theta_{0}, f \theta_{0}\right)}{1+d_{b}\left(\theta_{0}, f \theta_{0}\right)}, \frac{d_{b}\left(\theta_{1}, f \theta_{1}\right)}{1+d_{b}\left(\theta_{1}, f \theta_{1}\right)}, \frac{1}{2 s}\left[d_{b}\left(\theta_{0}, f \theta_{1}\right)+d_{b}\left(\theta_{1}, f \theta_{0}\right)\right]\right\} \\
& \leq \max \left\{d_{b}\left(\theta_{0}, \theta_{1}\right), d_{b}\left(\theta_{0}, f \theta_{0}\right), d_{b}\left(\theta_{1}, f \theta_{1}\right), \frac{1}{2 s} d_{b}\left(\theta_{0}, f \theta_{1}\right)\right\} \\
& \leq \max \left\{d_{b}\left(\theta_{0}, \theta_{1}\right), d_{b}\left(\theta_{0}, \theta_{1}\right), d_{b}\left(\theta_{1}, f \theta_{1}\right), \frac{1}{2}\left[d_{b}\left(\theta_{0}, \theta_{1}\right)+d_{b}\left(\theta_{1}, f \theta_{1}\right)\right]\right\} \\
& =\max \left\{d_{b}\left(\theta_{0}, \theta_{1}\right), d_{b}\left(\theta_{1}, f \theta_{1}\right)\right\} .
\end{aligned}
$$

If $\max \left\{d_{b}\left(\theta_{0}, \theta_{1}\right), d_{b}\left(\theta_{1}, f \theta_{1}\right)\right\}=d_{b}\left(\theta_{1}, f \theta_{1}\right)$, then by (5) and having in mind that $\psi(t)<t$ for each $t>0$, we get

$$
0<d_{b}\left(\theta_{1}, f \theta_{1}\right) \leq F\left(\psi\left(d_{b}\left(\theta_{1}, f \theta_{1}\right)\right), \phi\left(d_{b}\left(\theta_{1}, f \theta_{1}\right)\right)\right) \leq \psi\left(d_{b}\left(\theta_{1}, f \theta_{1}\right)\right)<d_{b}\left(\theta_{1}, f \theta_{1}\right),
$$

which is a contradiction. Thus, $\max \left\{d_{b}\left(\theta_{0}, \theta_{1}\right), d_{b}\left(\theta_{1}, f \theta_{1}\right)\right\}=d_{b}\left(\theta_{0}, \theta_{1}\right)$. Using again (5) and the fact that $\psi$ is nondecreasing, we get that

$$
0<d_{b}\left(\theta_{1}, f \theta_{1}\right) \leq \psi\left(d_{b}\left(\theta_{0}, \theta_{1}\right)\right)
$$

This implies that there exists $\theta_{2} \in f \theta_{1}$ (of course, $\theta_{2} \neq \theta_{1}$ ) such that

$$
0<d_{b}\left(\theta_{1}, \theta_{2}\right)<\psi\left(d_{b}\left(\theta_{0}, \theta_{1}\right)\right) .
$$

Since $\alpha\left(\theta_{0}, \theta_{1}\right) \geq s, \theta_{1} \in f \theta_{0}$ and $\theta_{2} \in f \theta_{1}$, using the fact that $f$ is $\alpha$-admissible, we get $\alpha\left(\theta_{1}, \theta_{2}\right) \geq s$. If $\theta_{2} \in f \theta_{2}, \theta_{2}$ is a fixed point of $f$. Otherwise, $\theta_{2} \notin f \theta_{2}$, so we have $d_{b}\left(\theta_{2}, f \theta_{2}\right)>0$. Applying again (3), we have

$$
\begin{aligned}
0<d_{b}\left(\theta_{2}, f \theta_{2}\right) & \leq H\left(f \theta_{1}, f \theta_{2}\right) \\
& \leq F\left(\psi\left(M\left(\theta_{1}, \theta_{2}\right)\right), \phi\left(M\left(\theta_{1}, \theta_{2}\right)\right)\right),
\end{aligned}
$$

where

$$
\begin{aligned}
M\left(\theta_{1}, \theta_{2}\right) & =\max \left\{d_{b}\left(\theta_{1}, \theta_{2}\right), \frac{d_{b}\left(\theta_{1}, f \theta_{1}\right)}{1+d_{b}\left(\theta_{1}, f \theta_{1}\right)}, \frac{d_{b}\left(\theta_{2}, f \theta_{2}\right)}{1+d_{b}\left(\theta_{2}, f \theta_{2}\right)}, \frac{1}{2 s}\left[d_{b}\left(\theta_{1}, f \theta_{2}\right)+d_{b}\left(\theta_{2}, f \theta_{1}\right)\right]\right\} \\
& \leq \max \left\{d_{b}\left(\theta_{1}, \theta_{2}\right), d_{b}\left(\theta_{1}, \theta_{2}\right), d_{b}\left(\theta_{2}, f \theta_{2}\right), \frac{1}{2 s} d_{b}\left(\theta_{1}, f \theta_{2}\right)\right\} \\
& \leq \max \left\{d_{b}\left(\theta_{1}, \theta_{2}\right), d_{b}\left(\theta_{1}, \theta_{2}\right), d_{b}\left(\theta_{2}, f \theta_{2}\right), \frac{1}{2 s}\left[d_{b}\left(\theta_{1}, \theta_{2}\right)+d_{b}\left(\theta_{2}, f \theta_{2}\right)\right]\right\} \\
& =\max \left\{d_{b}\left(\theta_{1}, \theta_{2}\right), d_{b}\left(\theta_{2}, f \theta_{2}\right)\right\} .
\end{aligned}
$$

Proceeding similarly as above, we get that $\max \left\{d_{b}\left(\theta_{1}, \theta_{2}\right), d_{b}\left(\theta_{2}, f \theta_{2}\right)\right\}=d_{b}\left(\theta_{1}, \theta_{2}\right)$. From (7) and (8),

$$
0<d_{b}\left(\theta_{2}, f \theta_{2}\right) \leq \psi\left(d_{b}\left(\theta_{1}, \theta_{2}\right)\right)<\psi^{2}\left(d_{b}\left(\theta_{0}, \theta_{1}\right)\right) .
$$

This implies again that there exists $\theta_{3} \in f \theta_{2}$ (of course, $\theta_{3} \neq \theta_{2}$ ) such that

$$
0<d_{b}\left(\theta_{2}, \theta_{3}\right)<\psi^{2}\left(d_{b}\left(\theta_{0}, \theta_{1}\right)\right) .
$$

Since $\alpha\left(\theta_{1}, \theta_{2}\right) \geq s, \theta_{2} \in f \theta_{1}$ and $\theta_{3} \in f \theta_{2}$, using the fact that $f$ is $\alpha$-admissible, we get $\alpha\left(\theta_{2}, \theta_{3}\right) \geq s$. If $\theta_{3} \in f \theta_{3}, \theta_{3}$ is a fixed point of $f$. Otherwise, $\theta_{3} \notin f \theta_{3}$, so we have $d_{b}\left(\theta_{3}, f \theta_{3}\right)>0$. Similarly, we get

$$
0<d_{b}\left(\theta_{3}, f \theta_{3}\right) \leq \psi\left(d_{b}\left(\theta_{2}, \theta_{3}\right)\right)<\psi^{3}\left(d_{b}\left(\theta_{0}, \theta_{1}\right)\right) .
$$


By continuing this process, we can construct a sequence $\left\{\theta_{n}\right\}$ in $X$ such that $\theta_{n} \notin f \theta_{n}, \theta_{n+1} \in f \theta_{n}$, $\alpha\left(\theta_{n}, \theta_{n+1}\right) \geq s$ and

$$
0<d_{b}\left(\theta_{n}, f \theta_{n}\right) \leq d_{b}\left(\theta_{n}, \theta_{n+1}\right) \leq \psi^{n}\left(d_{b}\left(\theta_{0}, \theta_{1}\right)\right), \forall n \in \mathbb{N} .
$$

Let $m, n \in \mathbb{N}$ be such that $m>n$. Then,

$$
d_{b}\left(\theta_{n}, \theta_{m}\right) \leq \sum_{i=n}^{m-1} s^{i-n+1} d_{b}\left(\theta_{i}, \theta_{i+1}\right) \leq \sum_{i=n}^{\infty} s^{i} \psi^{i}\left(d_{b}\left(\theta_{0}, \theta_{1}\right)\right) .
$$

Since $\psi \in \Psi,\left\{\theta_{n}\right\}$ is a Cauchy sequence in the complete $b$-metric space $\left(X, d_{b}\right)$. Hence, there exists $z \in X$ such that $z_{n} \rightarrow z$. Since $\theta_{n+1} \in f \theta_{n}$, we have $\left(\theta_{n}, \theta_{n+1}\right) \in G r(f)$. The graph is closed, so as $n \rightarrow \infty$, we get that $\left(\theta_{n}, \theta_{n+1}\right) \rightarrow(z, z)$, with $(z, z) \in G r(f)$. We deduce that $z \in f z$, that is, $z$ is a fixed point of $f$.

Now, we state the following known lemma.

Lemma 5 ([38]). Assume that $f x \in C L(X)$ for each $x \in X$. If $f$ is upper semi-continuous then $G r(f)$ is closed in $X^{2}$.

Following Lemma 5, we may provide the following result, even the upper semi-continuity condition is a strong hypothesis. Indeed, we may have a multivalued mapping such that its graph is closed, but it is not upper semi-continuous (see Reference [39]).

Theorem 2. Let $\left(X, d_{b}, s\right)$ be a $b$-metric space with a constant $s \geq 1$ and $f: X \rightarrow C L(X)$ be an $(\alpha, F)$-admissible multi-valued mapping. Assume that:

(i) $f$ is $\alpha$-admissible of type $S$;

(ii) there exist $\theta_{0} \in X$ and $\theta_{1} \in f \theta_{0}$ such that $\alpha\left(\theta_{0}, \theta_{1}\right) \geq s$;

(iii) $f$ is upper semi-continuous.

Then, $f$ has a fixed point.

It is also possible to omit the closedness of the graph of $f$ (or the upper semi-continuity of the mapping $f$ ) by introducing a regularity condition and adding the continuity of $\psi$.

Theorem 3. Let $\left(X, d_{b}, s\right)$ be a $b$-metric space with coefficient $s \geq 1$ and $f: X \rightarrow C L(X)$ be an $(\alpha, F)-$ multi-valued mapping. Assume that:

(i) $f$ is $\alpha$-admissible of type $S$ and $\psi$ is continuous;

(ii) there exist $\theta_{0} \in X$ and $\theta_{1} \in f \theta_{0}$ such that $\alpha\left(\theta_{0}, \theta_{1}\right) \geq s$;

(iii) if $\left\{\theta_{n}\right\}$ is a sequence in $X$ with $\theta_{n} \rightarrow u \in X$ and $\alpha\left(\theta_{n}, \theta_{n+1}\right) \geq$ s for all $n \in \mathbb{N} \cup\{0\}$, then $\alpha\left(\theta_{n}, u\right) \geq s$ for all $n \in \mathbb{N} \cup\{0\}$.

Then, $f$ has a fixed point.

Proof. Following the proof of Theorem 1, there exists a sequence $\left\{\theta_{n}\right\}$ such that

$$
\theta_{n+1} \in f \theta_{n}, \theta_{n} \notin f \theta_{n} \text { and } \alpha\left(\theta_{n}, \theta_{n+1}\right) \geq s \text { for all } n \in \mathbb{N} \cup\{0\},
$$

Furthermore, $\left\{\theta_{n}\right\}$, which is a Cauchy sequence in $X$, converges to some $u$ as $n \rightarrow \infty$. By Condition (iii), we get

$$
\alpha\left(\theta_{n}, u\right) \geq s \text { for all } n \in \mathbb{N} \cup\{0\} .
$$


If $u \in f u$, the proof is completed. We assume that $d_{b}(u, f u)>0$. We have

$$
\begin{aligned}
0<d_{b}(u, f u) & \leq s\left[d_{b}\left(u, \theta_{n+1}\right)+d_{b}\left(\theta_{n+1}, f u\right)\right] \\
& \leq s d_{b}\left(u, \theta_{n+1}\right)+s H\left(f \theta_{n}, f u\right) \\
& \leq s d_{b}\left(u, \theta_{n+1}\right)+s F\left(\psi\left(M\left(\theta_{n}, u\right)\right), \phi\left(M\left(\theta_{n}, u\right)\right)\right) \\
& \leq s d_{b}\left(u, \theta_{n+1}\right)+s \psi\left(M\left(\theta_{n}, u\right)\right)
\end{aligned}
$$

where

$$
\begin{aligned}
M\left(\theta_{n}, u\right) & =\max \left\{d_{b}\left(\theta_{n}, u\right), \frac{d_{b}\left(\theta_{n}, f \theta_{n}\right)}{1+d_{b}\left(\theta_{n}, f \theta_{n}\right)}, \frac{d_{b}(u, f u)}{1+d_{b}(u, f u)}, \frac{1}{2 s}\left[d_{b}\left(\theta_{n}, f u\right)+d_{b}\left(u, f \theta_{n}\right)\right]\right\} \\
& \leq \max \left\{d_{b}\left(\theta_{n}, u\right), d_{b}\left(\theta_{n}, \theta_{n+1}\right), d_{b}(u, f u), \frac{1}{2}\left[d_{b}\left(\theta_{n}, f u\right)+d_{b}\left(u, \theta_{n+1}\right)\right]\right\} \\
& \leq \max \left\{d_{b}\left(\theta_{n}, u\right), d_{b}\left(\theta_{n}, \theta_{n+1}\right), d_{b}(u, f u), \frac{1}{2}\left[d_{b}\left(\theta_{n}, f u\right)+d_{b}\left(u, \theta_{n+1}\right)\right]\right\} .
\end{aligned}
$$

Letting $n \rightarrow \infty$, we have $\lim \sup _{n \rightarrow \infty} M\left(\theta_{n}, u\right) \leq d_{b}(u, f u)$. Using the continuity of $\psi$, we get $\limsup _{n \rightarrow \infty} \psi\left(M\left(\theta_{n}, u\right)\right) \leq \psi\left(d_{b}(u, f u)\right)$. Letting $n \rightarrow \infty$ in (12), we obtain

$$
0<d_{b}(u, f u) \leq s \psi\left(d_{b}(u, f u)\right)<d_{b}(u, f u),
$$

which is a contradiction. Therefore, $u \in f u$ and hence $f$ has a fixed point.

Corollary 1. Let $\left(X, d_{b}, s\right)$ be a $b$-metric space with constant $s \geq 1$ and $f: X \rightarrow C L(X)$ be an $(\alpha, F)-$ multivalued mapping. Suppose there exist $\alpha: X \rightarrow[0, \infty), \psi \in \Psi$, and $\phi \in \Phi$ such that

$$
\alpha(x, y) H(f x, f y)) \leq F(\psi(M(x, y)), \phi(M(x, y))),
$$

for all $x, y \in X$. Assume that:

(i) $f$ is $\alpha$-admissible of type $S$;

(ii) there exist $\theta_{0} \in X$ and $\theta_{1} \in f \theta_{0}$ such that $\alpha\left(\theta_{0}, \theta_{1}\right) \geq s$;

(iii) the graph of $f$ is closed.

Then, $f$ has a fixed point.

Proof. By (14), the contraction (3) holds for all $x, y \in X$ with $\alpha(x, y) \geq s$. Thus, $f$ is an $(\alpha, F)-$ multi-valued mapping. By Theorem 1, the multi-valued mapping $f$ has a fixed point.

Corollary 2. Let $\left(X, d_{b}, s\right)$ be a $b$-metric space with a constant $s \geq 1$ and $f: X \rightarrow C L(X)$ be an $(\alpha, F)-$ multi-valued mapping. Assume that there exist $\alpha: X \rightarrow[0, \infty), \psi \in \Psi$, and $\phi \in \Phi$ such that

$$
\alpha(x, y) H(f x, f y)) \leq F(\psi(M(x, y)), \varphi(M(x, y))),
$$

for all $x, y \in X$. Assume that:

(i) $f$ is $\alpha$-admissible of type $S$ and $\psi$ is continuous;

(ii) there exist $\theta_{0} \in X$ and $\theta_{1} \in f \theta_{0}$ such that $\alpha\left(\theta_{0}, \theta_{1}\right) \geq s$;

(iii) $\left\{\theta_{n}\right\}$ is a sequence in $X$ with $\theta_{n} \rightarrow x \in X$ and $\alpha\left(\theta_{n}, \theta_{n+1}\right) \geq s$ for all $n \in \mathbb{N} \cup\{0\}$, then $\alpha\left(\theta_{n}, x\right) \geq s$ for all $n \in \mathbb{N} \cup\{0\}$.

Then, $f$ has a fixed point.

Proof. Again, by (15), the contraction (3) holds for all $x, y \in X$ such that $\alpha(x, y) \geq s$. Thus, $f$ is an $(\alpha, F)$-multi-valued mapping. By Theorem $3, f$ has a fixed point. 
By considering singlevalued mappings in Theorem 1 and Theorem 3, respectively, we have the following:

Corollary 3. Let $\left(X, d_{b}, s\right)$ be $a b$-metric space with coefficient $s \geq 1$ and $f: X \rightarrow X$. Suppose there exist $\alpha: X \times X \rightarrow[0,+\infty), F \in \mathcal{C}, \psi \in \Psi$, and $\phi \in \Phi$ such that

$$
\left.x, y \in X \text { with } \alpha(x, y) \geq s \Rightarrow d_{b}(f x, f y)\right) \leq F(\psi(M(x, y)), \varphi(M(x, y))),
$$

where $M(x, y)$ was defined by (4). Assume that:

(i) $u, v \in X, \alpha(u, v) \geq$ s implies $\alpha(f u, f v) \geq s$;

(ii) there exists $\theta_{0} \in X$ such that $\alpha\left(\theta_{0}, f \theta_{0}\right) \geq s$;

(iii) the graph of $f$ is closed.

Then, $f$ has a fixed point.

Corollary 4. Let $\left(X, d_{b}, s\right)$ be $a b$-metric space with coefficient $s \geq 1$ and $f: X \rightarrow X$. Suppose there exist $\alpha: X \times X \rightarrow[0,+\infty), F \in \mathcal{C}, \psi \in \Psi$, and $\phi \in \Phi$ such that the Condition (16) holds. Assume that:

(i) $u, v \in X, \alpha(u, v) \geq$ s implies $\alpha(f u, f v) \geq$ s. Also, $\psi$ is continuous;

(ii) there exists $\theta_{0} \in X$ such that $\alpha\left(\theta_{0}, f \theta_{0}\right) \geq s$;

(iii) if for $\xi_{0} \in X$, the sequence $\left\{\xi_{n}=f^{n} \xi_{0}\right\}$ in $X$ is such that $f^{n} \xi_{0} \rightarrow \xi \in X$ and $\alpha\left(f^{n} \xi_{0}, f^{n+1} \xi_{0}\right) \geq s$ for each integer $n \geq 0$, then $\alpha\left(f^{n} \xi_{0}, \xi\right) \geq$ s for each $n \geq 0$.

Then, $f$ has a fixed point.

Example 6. Let $X=[0, \infty)$ be endowed with the $b$-metric $d_{b}(x, y)=(x-y)^{2}$ with $s=2$ for all $x, y \in X$. Define $f: X \rightarrow C L(X)$ and $\alpha: X \rightarrow[0, \infty)$ by

$$
\begin{gathered}
f x= \begin{cases}{\left[0, \frac{x}{4}\right]} & \text { if } x \in[0,1] \\
{\left[x, x^{2}\right]} & \text { if } x \in(1, \infty)\end{cases} \\
\alpha(x, y)= \begin{cases}2 & \text { if } x, y \in[0,1] \\
0 & \text { otherwise. }\end{cases}
\end{gathered}
$$

Furthermore, define the functions $\psi, \phi:[0, \infty) \rightarrow[0, \infty)$ by $\psi(t)=t, \phi(t)=\frac{15}{16} t$. Take $F(r, t)=r-t$ for all $r, t \in[0, \infty)$.

Firstly, we show that $f$ is $\alpha$-admissible of type $S$. Let $x \in X$ and $y \in f x$ with $\alpha(x, y) \geq s=2$. Then, $x, y \in[0,1]$. Let $z \in f y$, then $z \in\left[0, \frac{y}{4}\right] \subset\left[0, \frac{x}{16}\right] \subset[0,1]$. Then,

$$
\alpha(y, z)=2=s
$$

Hence, $f$ is $\alpha$-admissible of type $S$. For $\theta_{0}=\frac{1}{2}$ and $\theta_{1}=\frac{1}{16} \in f \theta_{0}$, we have $\alpha\left(\theta_{0}, \theta_{1}\right)=2=$ s. For any sequence $\left\{\theta_{n}=f^{n} \theta_{0}\right\} \subseteq X$ (where $\theta_{0} \in X$ is arbitrary) such that $\theta_{n} \rightarrow x$ as $n \rightarrow \infty$ and $\alpha\left(\theta_{n}, \theta_{n+1}\right)=2=s$ for each $n \in \mathbb{N}$, we have $x_{n}, x \in[0,1]$, so $\alpha\left(\theta_{n}, x\right)=2=$ s for each $n \in \mathbb{N}$.

Now, we will show that the conditions of Theorem 3 are fulfilled for all $x, y \in X$ with $\alpha(x, y) \geq s$, that is, $x, y \in[0,1]$. Without loss of generality, take $x \neq y$. Then,

$$
H(f x, f y)=\frac{(x-y)^{2}}{16}
$$

Thus,

$$
H(f x, f y)=\frac{(x-y)^{2}}{16}=\frac{1}{16} d_{b}(x, y) \leq \frac{1}{16} M(x, y)=F(\psi(M(x, y)), \phi(M(x, y)))
$$

All hypotheses of Theorem 3 are satisfied and $f$ has a fixed point. Here, 0 and each $x>1$ are fixed points of $f$. 
Note also that the graph of $f$ is closed, so we may also apply Theorem 1.

\section{Application}

The aim of this section is to give an application on the existence of a solution for an integral equation by applying Corollary 4 .

First, let $X$ be the set of continuous functions specified on the closed interval $[a, b]$. We endow $X$ by the standard $b$-metric $d_{b}: X \times X \rightarrow[0, \infty)$ :

$$
d_{b}(x, y)=\left(\sup _{t \in[a, b]}|x(t)-y(t)|\right)^{2},
$$

for all $x, y \in X$. Then, $\left(X, d_{b}\right)$ is a complete $b-$ metric space with coefficient $s=2$.

We consider the following integral equation:

$$
y(r)=y_{0}+\int_{a}^{b} P(r, t) g(t, y(t)) d t
$$

where $y_{0} \in \mathbb{R}$ and $P:[a, b] \times[a, b] \rightarrow[0, \infty), g:[a, b] \times \mathbb{R} \rightarrow \mathbb{R}$ are continuous functions.

Define $f: X \rightarrow X$ as

$$
f y(r)=y_{0}+\int_{a}^{b} P(r, t) g(t, y(t)) d t .
$$

Then, a solution of Equation (17) is equivalent to stating that the map $f$ has a fixed point.

Now, we will prove the following result applying Corollary 4.

Theorem 4. Assume that the following conditions are satisfied:

(i) there exists $\alpha: X \times X \rightarrow[0, \infty)$ such that if $\alpha(u, v) \geq s=2$ for $u, v \in X$, we have for each $t \geq 0$,

$$
\mid g\left(t, u(t)-g\left(t, v(t) \mid \leq \sqrt{\ln \left(1+(|u(t)-v(t)|)^{2}\right)},\right.\right.
$$

and

$$
\sup _{r \in[a, b]} \int_{a}^{b} P(r, t) d t \leq 1
$$

(ii) $u, v \in X, \alpha(u, v) \geq 1$ implies $\alpha(f u, f v) \geq 1$;

(iii) there exists $u_{0} \in X$ such that $\alpha\left(u_{0}, f u_{0}\right) \geq s=2$;

(iv) if $\left\{\xi_{n}=: f^{n} \xi_{0}\right\}$ (where $\xi_{0}$ is arbitrary in $X$ ) is a sequence in $X$ with $\xi_{n} \rightarrow \xi \in X$ and $\alpha\left(\xi_{n}, \xi_{n+1}\right) \geq s$ for each integer $n \geq 0$, then $\alpha\left(\xi_{n}, \xi\right) \geq$ s for each $n \geq 0$.

Then, the integral Equation (17) has a solution in X.

Proof. For all $u, v \in X$, we have

$$
\left.d_{b}(f u, f v)=\sup _{r \in[a, b]}|f u(r)-f v(r)|\right)^{2} .
$$


We also have for $r \in[a, b]$,

$$
\begin{aligned}
& (|f u(r)-f v(r)|)^{2} \\
= & \left(\left|\int_{a}^{b} P(r, t)\right| g(t, u(t))-g(t, v(t))|d t|\right)^{2} \\
\leq & \left(\int_{a}^{b} P(r, t)|g(t, u(t))-g(t, v(t))| d t\right)^{2} \\
\leq & \left(\int_{a}^{b} P(r, t) \sqrt{\ln \left(1+(|u(t)-v(t)|)^{2}\right)} d t\right)^{2} \\
\leq & \left(\int_{a}^{b} P(r, t) \sqrt{\ln \left(1+d_{b}(u, v)\right)} d t\right)^{2} \\
\leq & \left(\int_{a}^{b} P(r, t) d t\right)^{2} \ln \left(1+d_{b}(u, v)\right) \\
\leq & \ln \left(1+d_{b}(u, v)\right) .
\end{aligned}
$$

Thus,

$$
\begin{aligned}
H(f u, f v) & \leq \ln \left(1+d_{b}(u, v)\right) \leq \ln (1+M(u, v)) \\
& =M(u, v)-(M(u, v)-\ln (1+M(u, v))) \\
& =F(\psi(M(u, v)), \phi(M(u, v)))
\end{aligned}
$$

where $\psi(t)=t$ (it is continuous), $\phi(t)=t-\ln (1+t)$, and $F(s, t)=s-t$. Since (iii) and (iv) hold, all hypotheses of Corollary 4 hold. Thus, $f$ has a fixed point, that is, the integral Equation (17) has a solution in $X$.

\section{Conclusions}

Many researchers proved the existence and uniqueness of some fixed point results for self-mappings in b-metric spaces. In this paper, we have introduced the existence and uniqueness of fixed point results for $(\alpha, \psi, \phi)$-admissible multi-valued mappings in the setting of $b$-metric spaces by defining a weak contractive condition using $C$ - functions and $\alpha$-admissible multi-valued mappings of type $S$. We have provided examples and an application of integral equations to show the superiority of our results and given concepts. Furthermore, as perspectives, we suggest extending more results to find (common) fixed point results in generalized metric spaces as extended $b$-metric spaces, $b$-metric-like spaces. Otherwise, we recommend utilizing our main results for non-self-mappings to establish the existence of an optimal approximate solution.

Author Contributions: All authors contributed equally and significantly in writing this article. All authors read and approved the final manuscript.

Funding: The authors would like to thank Universiti Kebangsaan Malaysia for supporting this paper through Grant GP-K005224 and Ministry of Education, Malaysia grant FRGS/1/2017/STG06/UKM/01/1.

Acknowledgments: The authors would like to thank the anonymous reviewers and editor for their valuable remarks on our paper.

Conflicts of Interest: The authors declare no conflict of interest. 


\section{Abbreviations}

MDPI Multidisciplinary Digital Publishing Institute

DOAJ Directory of open access journals

TLA Three letter acronym

LD linear dichroism

\section{References}

1. Aydi, H.; Shatanawi, W.; Vetro, C. On generalized weak G-contraction mapping in G-metric spaces. Comput. Math. Appl. 2011, 62, 4223-4229. [CrossRef]

2. Dukic, D.; Kadelburg, Z.; Radenovic, S. Fixed point of Geraghty-type mappings in various generalized metric spaces. Abstr. Appl. Anal. 2011. [CrossRef]

3. Aydi, H.; Bota, M.F.; Karapinar, E.; Moradi, S. A common fixed point for weak $\phi$-contractions on b-metric spaces. Fixed Point Theory 2012, 13, 337-346.

4. Aydi, H.; Felhi, A.; Sahmim, S. Common fixed points via implicit contractions on $b$-metric-like spaces. J. Nonlinear Sci. Appl. 2017, 10, 1524-1537. [CrossRef]

5. Qawaqneh, H.; Noorani, M.S.M.; Shatanawi, W.; Alsamir, H. Common fixed points for pairs of triangular $\alpha$-admissible mappings. J. Nonlinear Sci. Appl. 2017, 10, 6192-6204. [CrossRef]

6. Qawaqneh, H.; Noorani, M.S.M.; Shatanawi, W.; Abodayeh, K.; Alsamir, H. Fixed point for mappings under contractive condition based on simulation functions and cyclic $(\alpha, \beta)$-admissibility. J. Math. Anal. 2018, 9 , 38-59. [CrossRef]

7. Qawaqneh, H.; Noorani, M.S.M.; Shatanawi, W. Fixed Point Results for Geraghty Type Generalized F-contraction for Weak $\alpha$-admissible Mapping in Metric-like Spaces. Eur. J. Pure Appl. Math. 2018, 11, 702-716. [CrossRef]

8. Qawaqneh, H.; Noorani, M.S.M.; Shatanawi, W.; Alsamir, H. Common Fixed Point Theorems for Generalized Geraghty $(\alpha, \psi, \phi)$-Quasi Contraction Type Mapping in Partially Ordered Metric-like Spaces. Axioms 2018, 7, 74. [CrossRef]

9. Mlaiki, N.; Aydi, H.; Souayah, N.; Abdeljawad, T. Controlled metric type spaces and the related contraction principle. Mathematics 2018, 6, 194. [CrossRef]

10. Qawaqneh, H.; Noorani, M.S.M.; Shatanawi, W. Fixed Point Theorems for $(\alpha, k, \theta)$-Contractive Multi-Valued Mapping in $b$-Metric Space and Applications. Int. J. Math. Comput. Sci. 2019, 14, 263-283.

11. Ciric, L.B.; Samet, B.; Aydi, H.; Vetro, C. Common fixed points of generalized contractions on partial metric spaces and an application. Appl. Math. Comput. 2011, 218, 2398-2406.

12. Meir, A.; Keeler, E. A theorem on contraction mappings. J. Math. Anal. Appl. 1969, 28, 326-329. [CrossRef]

13. Shatanawi, W.; Postolache, M. Common fixed point results for mappings under nonlinear contraction of cyclic form in ordered metric spaces. Fixed Point Theory Appl. 2013, 2013. [CrossRef]

14. Nadler, J. Multi-valued contraction mappings. Pac. J. Math. 1969, 30, 475-488. [CrossRef]

15. Aydi, H.; Abbas, M.; Vetro, C. Partial Hausdorff metric and Nadler's fixed point theorem on partial metric spaces. Topol. Appl. 2012, 159, 3234-3242. [CrossRef]

16. Berinde, V.; Pacurar, M. The role of the Pompeiou Hausdorff metric in fixed point theory. Creat. Math. Inform. 2013, 22, 143-150.

17. De la Sen, M.; Singh, S.L.; Gordji, M.E.; Ibeas, A.; Agarwal, R.P. Best proximity and fixed point results for cyclic multivalued mappings under a generalized contractive condition. Fixed Point Theory Appl. 2013, 2013, 324. [CrossRef]

18. Bakhtin, I.A. The contraction mapping principle in almost metric spaces. Funct. Anal. 1989, 30, $26-37$.

19. Czerwik, S. Contraction mappings in b-metric spaces. Acta Math. Inform. Univ. Ostrav. 1993, 30, 5-11.

20. Aydi, H.; Karapinar, E.; Bota, M.F.; Mitrović, S. A fixed point theorem for set-valued quasi-contractions in b-metric spaces. Fixed Point Theory Appl. 2012, 88. [CrossRef]

21. Czerwick, S.; Dlutek, K.; Singh, S.L. Round-off stability of iteration procedures for set-valued operators in $b$-metric spaces. J. Nat. Phys. Sci. 2001, 11, 87-94.

22. Karapinar, E.; Czerwik, S.; Aydi, H. $(\alpha, \psi)$-Meir-Keeler contraction mappings in generalized b-metric spaces. J. Funct. Spaces 2018, 2018. [CrossRef] 
23. Qawaqneh, H.; Noorani, M.S.M.; Shatanawi, W.; Alsamir, H. Some fixed point results for the cyclic $(\alpha, \beta)-$ $(k, \theta)$-multi-valued mappings in metric space. J. Phys. Conf. Ser. 2018. [CrossRef]

24. Samet, B.; Vetro, C.; Vetro, P. Fixed point theorems for $\alpha, \psi$-contractive type mappings. Nonlinear Anal. 2012, 75, 2154-2165. [CrossRef]

25. Sintunavarat, W. Nonlinear integral equations with new admissibility types in b-metric spaces. J. Fixed Point Theory Appl. 2016, 18, 397-416. [CrossRef]

26. Jleli, M.; Samet, B.; Vetro, C.; Vetro, F. Fixed Points for Multi-valued Mappings in $b$-Metric Spaces. Abstr. Appl. Anal. 2015, 2015. [CrossRef]

27. Felhi, A.; Aydi, H.; Zhang, D. Fixed points for $\alpha$-admissible contractive mappings via simulation functions. J. Nonlinear Sci. Appl. 2016, 9, 5544-5560. [CrossRef]

28. Karapinar, E.; Samet, B. Generalized $\alpha-\psi$-contractive type mappings and related fixed point theorems with applications. Abstr. Appl. Anal. 2012, 2012. [CrossRef]

29. Ali, M.U.; Kamran, T.; Karapinar, E. $(\alpha, \psi, \xi)$-contractive multi-valued mappings. Fixed Point Theory Appl. 2014, 7. [CrossRef]

30. Karapinar, E.; Kumam, P.; Salimi, P. On $\alpha, \psi$-Meir-Keeler contractive mappings. Fixed Point Theory Appl. Abstr. Appl. Anal. 2013, 2013. [CrossRef]

31. Mohammadi, B.; Rezapour, S.; Shahzad, N. Some results on fixed points of $\alpha-\psi-$ Ciric generalized multifunctions. Fixed Point Theory Appl. 2013, 2013. [CrossRef]

32. Ansari, A.H. Note on $\varphi-\psi$-contractive type mappings and related fixed point. In Proceedings of the 2nd Regional Conference on Mathematics and Applications, Jyvaskyla, Finland, 18-19 September 2014; pp. 377-380. [CrossRef]

33. Fisher, B. On a theorem of Khan. Riv. Math. Univ. Parma 1978, 4, 1-4.

34. Aydi, H.; Postolache, M.; Shatanawi, W. Coupled fixed point results for $(\psi, \phi)$-weakly contractive mappings in ordered G-metric spaces. Comput. Math. Appl. 2012, 63, 298-309. [CrossRef]

35. Aydi, H.; Wongyat, T.; Sintunavarat, W. On new evolution of Ri's result via $w$-distances and the study on the solution for nonlinear integral equations and fractional differential equations. Adv. Differ. Equ. 2018, 32. [CrossRef]

36. Aydi, H. On common fixed point theorems for $(\psi, \varphi)$-generalized f-weakly contractive mappings. Miskolc Math. Notes 2013, 14, 19-30. [CrossRef]

37. Mustafa, Z.; Jaradat, M.M.M.; Aydi, H.; Alrhayyel, A. Some common fixed points of six mappings on $G_{b^{-}}$metric spaces using (E.A) property. Eur. J. Pure Appl. Math. 2018, 11, 90-109, doi.org/10.29020/nybg.ejpam.v11i1.3133. [CrossRef]

38. Hu, S.; Papageorgiou, N.S. Theory. In Handbook of Multivalued Analysis, 1st ed.; Kluwer Academic Publishers: Dordrecht, The Netherlands, 1997; Volume I.

39. Durmaz, G.; Altun, I. Fixed point results for $\alpha$-admissible mmutivalued F-contrtactions. Miscolc Math. Notes 2016, 17, 187-199. [CrossRef]

(C) 2019 by the authors. Licensee MDPI, Basel, Switzerland. This article is an open access article distributed under the terms and conditions of the Creative Commons Attribution (CC BY) license (http:/ / creativecommons.org/licenses/by/4.0/). 